\title{
Consideration of Nebulized Lidocaine For Treatment of Covid19 Severity Via Targeting Neutrophil Extracellular Traps
}

\author{
Raed Madhi* \\ Department of Clinical Sciences, Section of Surgery Malmö, Lund University, Sweden
}

*Corresponding author: Raed Madhi, Department of Clinical Sciences, Section of Surgery Malmö, Lund University, Sweden.

\begin{abstract}
Coronavirus disease 2019 (Covid19) is a viral-provoked respiratory disease that affects many patients worldwide. Clinical manifestations start with mild flu-like symptoms and progress to acute respiratory distress syndrome (ARDS), the trigger of which has been underpinned as cytokine storm. Neutrophil-derived neutrophil extracellular traps (NETs) greatly contribute to development of Covid19 severity. It has been hypothesized that nebulized lidocaine could be used to attenuate the severity of Covid19, however, the mechanism by which this may occur not completely clear. This review addresses the possibility of using nebulized lidocaine as a therapeutic approach for targeting Covid19-induced NETs formation.

Keywords: Covid19; Cytokines; Neutrophil; Neutrophil extracellular traps; Lung damage; Nebulized lidocaine

Abbreviations: Covid19-Coronavirus disease 2019; ARDS-Acute Respiratory Distress Syndrome; NETs-Neutrophil Extracellular Traps; SARSCoV-2-Severe Acute Respiratory Syndrome Coronavirus 2; ACE2-9-Angiotensin Converting Enzyme 2; G-CSF-Granulocyte Colony Stimulating Factor; MCP1-Monocyte Chemoattractant Protein 1; STAT3-Signal Transducer And Activator of Transcription 3; CF Cystic Fibrosis; NE-Neutrophil Elastase
\end{abstract}

\section{Introduction}

The WHO (World health organization) has declared that coronavirus disease 2019 (Covid19) is a disease of pandemic proportion that causes severe acute respiratory syndrome coronavirus 2 (SARS-CoV-2) [1]. It well known that lung is the most common organ infected by Covid19 and this could be due to high expression of angiotensin-converting enzyme 2 (ACE2) receptor in lung tissue [2,3]. The severity of Covid19 has been shown to be associated with elevated viscus mucus secretions in the airways, pro-inflammatory cytokine production, leukocyte recruitment and extreme lung damage. Because of the difficulty in management of this disease at late stage, it has induced fatality a large number of patients [4-6]. Serval studies have observed neutrophil infiltration increases in the lungs during Covid19 infection [7,8]. And it has been suggested that neutrophilia are a source of excess NETs and the hallmarks of acute infection of Covid19 [6]. In this review, I hypothesize that targeting Covid19-induced NETs by nebulized lidocaine could be a useful strategy for reducing the severity of Covid19 in patients and for decreasing the overall associated mortality rate.

\section{NETs and Cytokines Production}

The severity of Covid19 has been shown to be associated with cytokine production. For instance, it was observed that levels of TNF $\alpha$, MCP1, G-CSF, IL17, IL10, IL8, IL7, IL2, IL1 $\beta$ are elevated in 
the plasma of patients with Covid19 [9-13]. In fact, these cytokines serve as inflammatory mediators that mediate neutrophil activation and chemoattractant production. Neutrophil extravasation into inflamed tissue has also been documented to be mediated by these chemoattractant $[14,15]$. NETs can activate macrophages to secrete TNF $\alpha$, which in turn, enhances the release of NETs in different diseases, including lung inflammation and arthritis [16]. Indeed, infection with Covid19 has been shown to increase the levels of TNF $\alpha$ [17] and it has been suggested this may lead to accelerated formation of NETs (Figure1). A recent study has found that NETs can regulate transcription factors such as signal transducer and activator of transcription 3 (STAT3) [18]. This transcription factor has been found to control the expression of a verity of genes involved in onset of cytokine storm which is capable of exuberating pulmonary injury. Moreover, it was reported that NETs formation is associated with increased levels of plasma IL-6 [19]. This proinflammatory cytokine was found to mediate STAT3 activation [20]. Taken together these findings strongly suggest targeting these pro-inflammatory cytokines (TNF $\alpha$ and IL-6) could be involved in decreasing NETs formation in severe Covid19.

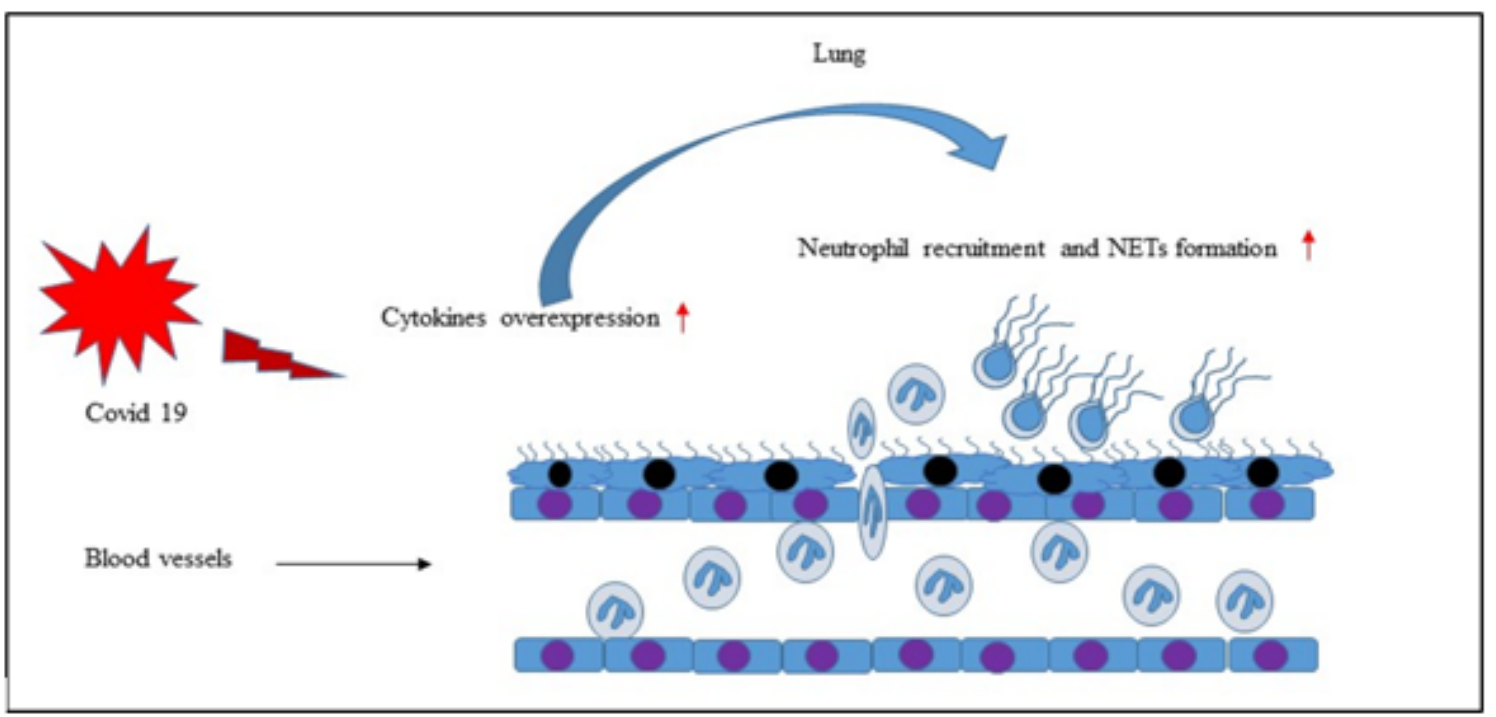

Figure 1: Basic illustration of Covid19 and NETs formation in Lung. Infection with Covid19 triggers hyper production of cytokines. Proinflammatory cytokines cause extravascular navigation of neutrophil in Lung tissue. Activated neutrophils expel NETs into lung tissue.

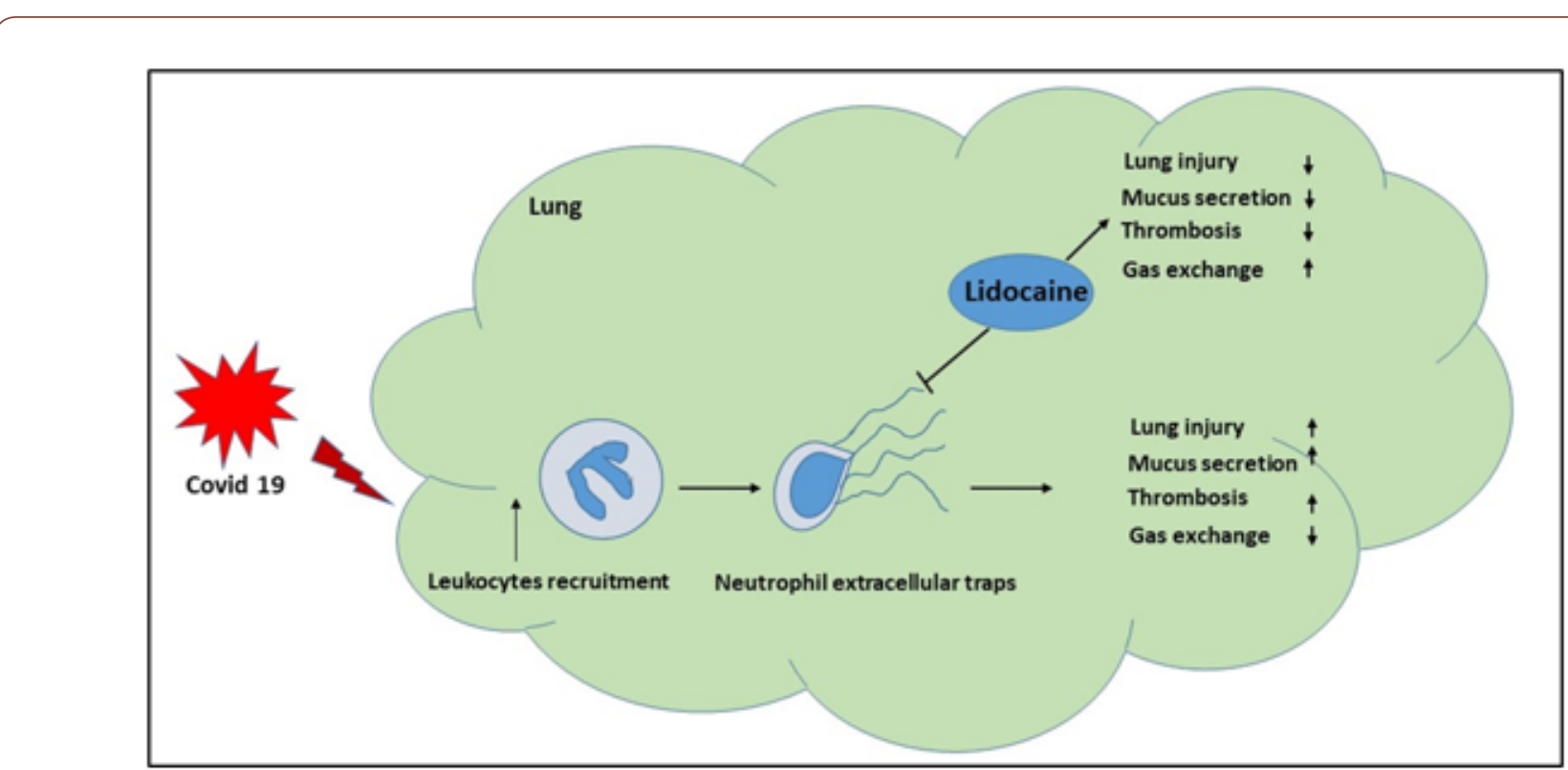

Figure 2: Basic abstract of inhibitory mechanism of lidocaine in Covid19. Covid19-induced NETs provoke thrombosis, viscus mucus secretions and lung damage that can led to decrease gas exchange. Nebulized lidocaine could protect against thrombosis, viscus mucus secretions and lung damage via possibly block NETs formation. 


\section{NETs and Leukocyte Recruitment}

During their activation, neutrophils have been observed to expel extracellular traps against invading pathogens [21]. However, disorder of NETs clearance or excessive NETs formation have been found to participate in tissue damage in both non-infectious diseases and infectious diseases [14,22]. The direct role of NETs in leukocyte recruitment has not examined, however, numerous studies have shown indirect evidence in which inhibiting or disintegrating NET formation was found to reduce the recruitment of inflammatory cells in models of inflammation [15,22]. Leukocyte extravasation is a gradual multistep process mediated by adhesion molecules expressed on both leukocytes and endothelial cells [23]. Accumulating data report that the selectin family of adhesion molecules (P, E, and L-selectin) provide a crucial support of leukocyte rolling via binding with their glycoprotein ligands [24, 25]. A study by Wang Y, et al. [26], has shown that NETs stimulate neutrophil rolling, adhesion, and extravasation in the microvasculature of cremaster tissue. The authors found that immunoneutralization of selectins, such as P-selectin, greatly attenuated NET-triggered neutrophil rolling and emigration [26]. Indicating that NETs cause self-amplifying loop via increase neutrophilia and chemotaxis. Indeed, Covid19 has been documented to elevate NETs formation in sera of patients with Covid19. Moreover, a recent study has been observed that incubation of healthy neutrophil with Covid19 patients' sera results in triggering NETs release form control neutrophil [27]. And NETs formation has been shown to positively provoke neutrophilia and neutrophil extravasation into lung tissue [28].

\section{NETs in Pulmonary Damage}

Accumulating data have reported that NETs formation is extensively linked to pulmonary diseases. Lingtao Luo et al. [22], have observed that NETs control pulmonary extravasation of neutrophil and tissue damage via regulating pro-inflammatory compounds in septic lung injury [22]. A previous study has found that NETs cause direct damage to epithelial and endothelial cells possibly via histone proteins, a major component of NETs [29]. Indeed, it was documented that levels of NETs are increased in the plasma of patients with acute respiratory distress syndrome (ARDS) [30]. Moreover, extracellular histones have been observed to increase in both plasma and bronchoalveolar lavage fluid of patients with ARDS [31]. Experimental studies have shown that NETs are involved in the development of ARDS and that either preventing production of or destruction of the same may protect against lung damage and increase survival [30,32-34]. Yongzhi Wang, et al. [35], found that NETs are dependent on thrombin formation and that dissolution of NETs formation antagonized sepsis-induced thrombin generation [35]. It has also shown that ARDS, sepsis, and multi-organ dysfunction syndrome are the most common complications of Covid19 [36,37], and thus targeting NETs is a potentially novel therapeutic strategy for attenuating the severity of Covid19.

\section{Nets in Cystic Fibrosis}

The severity of cystic fibrosis (CF) is attributed to accumulation of mucous purulent secretions in the airways. A previous study has observed that viscosity of these purulent secretions increases greatly in the presence of large amounts of extracellular DNA, a major component of NETs. The authors found that disintegration of extracellular DNA in purulent sputum of patients with $\mathrm{CF}$ reduced the viscosity and transformed the sputum from the gel-like structures to flowing liquid within minutes [38]. Neutrophil elastase (NE) has been shown to be one of the most granular proteins that constitutes NETs [39]. Overexpression of NETs with increased NE can elevate the viscosity and mucus thickness of purulent secretions in the airways of cystic CF fluid. Accordingly, mucus secretions have been reported to impair gas exchange as well as facilitate bacterial colonization in the lungs [40]. Consequently, colonization of bacteria can further stimulate neutrophil extravasation and recruitment as well as NET formation, and this in turn may increase the viscosity of secretions in the airways and cause respiratory dysfunction. Indeed, patients with Covid19 have been found to have mucous secretions in the airways of the lungs [41] and these secretions seem to be similar to those seen in patients with CF. Therefore, if the secretions of Covid19 patients contain NETs, then it is possible they play a similar role in this disease to that which they play in CF (i.e. encourage/induce respiratory dysfunction and facilitate bacterial colonization in the lungs).

\section{Lidocaine and Covid19-induced NETs formation}

Lidocaine or xylocaine is a chemical compound that causes local anesthesia via blocking nerve signals. Consequently, neurons consistently become unable to transmit signals to the brain. This medication was first introduced in 1946 as an anesthetic which can be administrated via many differing approaches, e.g. intravenous, subcutaneous, topical, and oral. Lidocaine can provide sedation starting- within minutes and lasting for up to several hours [42].

Indeed, lidocaine has recently been used to treat patients with acute asthma. It has been shown that nebulized inhalation of lidocaine can greatly reduce bronchial hypersensitivity in patients with acute asthma [43]. Caracas HC, et al. [44], have reported that lidocaine has a potential anti-inflammatory role in infectious and non-infectious diseases [44]. Moreover, a previous study has investigated the effectiveness-of lidocaine on leukocyte recruitment in surgical wounds in animal models. The authors have observed that treatment with lidocaine greatly attenuated the migration of leukocytes in surgical wounds [45]. Pro-inflammatory cytokines have been shown to stimulate the recruitment of leukocytes into the inflamed sites $[46,47]$. In fact, an experimental study has found that administration of lidocaine through both inhalation and intravenous injection resulted in a significant reduction of IL-6, TNF- $\alpha$ and IL-1 $\beta$ in lung tissue [48]. Moreover, it 
was also documented that intravenous administration of lidocaine greatly increased levels of the anti-inflammatory cytokine IL-10, in mechanically ventilated animal lungs [49]. In addition, clinical studies have found that receiving lidocaine can attenuate levels of pro-inflammatory cytokines $[50,51]$. Thus, the anti-inflammatory effect of lidocaine on cytokine production could in part explain the inhibitory effect of leukocyte migration.

It is well established that mucus clearance is an important mechanism for lung health and that disorder of this mechanism may led to disease of airway. Healthy mucus secretion is characterized as secretion of a gel-like substance of low viscosity and elasticity which can easily be transported through ciliary action. However, it has been demonstrated that in certain pulmonary disease pathologies the viscosity and elasticity of mucus is increased, and this leads to a subsequent decrease in mucus clearance that is debilitating for pulmonary function $[52,53]$. Moreover, it has been shown that the mucus of cystic fibrosis patients contains infiltrated neutrophils with high concentrations of neutrophil-derived extracellular DNA and actin filaments [54-56]. Another study has also documented that NETs formation represents the essential extracellular component of CF [40] and a recent study in a murine model of asthma has found that nebulized lidocaine attenuates airway inflammation and peribronchial fibrosis as well as mucus overproduction [57]. In addition, Elena et al, 2020 have been found that administration of lidocaine greatly attenuated NETosis in patients with breast cancer [58]. Importantly, the severity of Covid19 has been shown to be characterized by elevated neutrophil infiltration and mucous secretions in the airways of the lungs [28]. Furthermore, it was found that NETs formation increased in the sera of patients with Covid19 and that NETs can be used as a marker for the severity of Covid19 [27].

In this review, I highlighted that in addition to providing a local sedation, nebulized lidocaine could potentially be used as a future therapeutic approach for targeting Covid19-induced NETs formation and by this means protect against viscus mucus secretion and airway inflammation to protect/enhance lung function in Covid19 patients (Figure 2). Thus, future studies are warranted to investigate the possibility of nebulized lidocaine in targeting Covid19-induced NETs in patients with Covid19.

\section{Acknowledgment}

Swedish Medical Research Council (2017-01621) and Einar and Inga Nilsson foundation. Raed Madhi is supported by Misan University, College of Science, Iraq. I am grateful to Henrik Thorlacius, Milladur Rahman and Elaine Cowan.

\section{Conflict of Interest}

No conflict of interest.

\section{References}

1. Cucinotta D, Vanelli M (2020) WHO Declares COVID-19 a Pandemic. Acta Biomed 91(1): 157-160.
2. Gu J, Korteweg C (2007) Pathology and pathogenesis of severe acute respiratory syndrome. Am J Pathol 170(4): 1136-1147.

3. Kuba K, Imai Y, Penninger JM (2006) Angiotensin-converting enzyme 2 in lung diseases. Curr Opin Pharmacol 6(3): 271-276.

4. Wang D, Hu B, Hu C, Zhu F, Liu X, et al. (2020) Clinical Characteristics of 138 Hospitalized Patients With 2019 Novel Coronavirus-Infected Pneumonia in Wuhan, China. JAMA 323(11): 1061-1069.

5. Zheng M, Gao Y, Wang G, Song G, Liu S, et al. (2020) Functional exhaustion of antiviral lymphocytes in COVID-19 patients. Cell Mol Immunol 17(5): 533-535.

6. Barnes BJ, Adrover JM, Baxter Stoltzfus A, Borczuk A, Cools Lartigue J, et al. (2020) Targeting potential drivers of COVID-19: Neutrophil extracellular traps. J Exp Med 217(6): e20200652.

7. Fox S, Akmatbekov A, Harbert J, Li G, Brown Q et al. (2020) Pulmonary and Cardiac Pathology in Covid-19: The First Autopsy Series from New Orleans. medRxiv.

8. Yao XH, Li TY, He ZC, Ping YF, Liu HW, et al. (2020) A pathological report of three COVID-19 cases by minimal invasive autopsies. Zhonghua Bing Li Xue Za Zhi 49(5): 411-417.

9. Huang C, Wang Y, Li X, Ren L, Zhao J, et al. (2020) Clinical features of patients infected with 2019 novel coronavirus in Wuhan, China. Lancet 395(10223): 497-506.

10. Mehta P, Mc Auley DF, Brown M, Sanchez E, Tattersall RS, et al. (2020) COVID-19: consider cytokine storm syndromes and immunosuppression. Lancet 395(10229): 1033-1034.

11. Ruan Q, Yang K, Wang W, Jiang L, Song J (2020) Clinical predictors of mortality due to COVID-19 based on an analysis of data of 150 patients from Wuhan, China. Intensive Care Med 46(5): 846-848.

12. Wu C, Chen X, Cai Y, Xia J, Zhou X, et al. (2020) Risk Factors Associated With Acute Respiratory Distress Syndrome and Death in Patients With Coronavirus Disease 2019 Pneumonia in Wuhan, China. JAMA Intern Med 180(7): 934-943.

13. Zhang W, Zhao Y, Zhang F, Wang Q, Li T, et al (2020) The use of antiinflammatory drugs in the treatment of people with severe coronavirus disease 2019 (COVID-19): The Perspectives of clinical immunologists from China. Clin Immunol 214: 108393

14. Madhi R, Rahman M, Taha D, Morgelin M, Thorlacius H (2019) Targeting peptidylarginine deiminase reduces neutrophil extracellular trap formation and tissue injury in severe acute pancreatitis. J Cell Physiol 234(7): 11850-11860.

15. Madhi R, Rahman M, Morgelin M, Thorlacius H (2019) c-Abl kinase regulates neutrophil extracellular trap formation, inflammation, and tissue damage in severe acute pancreatitis. J Leukoc Biol 106(2): 455466.

16. Bawadekar M, Gendron Fitzpatrick A, Rebernick R, Shim D, Warner $\mathrm{TF}$, et al. (2016) Tumor necrosis factor alpha, citrullination, and peptidylarginine deiminase 4 in lung and joint inflammation. Arthritis Res Ther 18(1): 173.

17. Wu D, Yang XO (2020) TH17 responses in cytokine storm of COVID-19: An emerging target of JAK2 inhibitor Fedratinib. J Microbiol Immunol Infect 53(3): 368-370

18. Merza M, Hartman H, Rahman M, Hwaiz R, Zhang E, et al. (2015) Neutrophil Extracellular Traps Induce Trypsin Activation, Inflammation, and Tissue Damage in Mice With Severe Acute Pancreatitis. Gastroenterology 149(7): 1920-1931.

19. Barbu EA, Mendelsohn L, Samsel L, Thein SL (2020) Pro-inflammatory cytokines associate with NETosis during sickle cell vaso-occlusive crises. Cytokine 127: 154933.

20. Wang Y, Anette HH van Boxel Dezaire, Cheon H, Yang J, Stark GR (2013) STAT3 activation in response to IL- 6 is prolonged by the binding of IL- 6 receptor to EGF receptor. Proc Natl Acad Sci U S A 110(42): 1697516980. 
21. Brinkmann V, Reichard U, Goosmann C, Fauler B, Uhlemann Y, et al (2004) Neutrophil extracellular traps kill bacteria. Science 303(5663): $1532-1535$.

22. Luo L, Zhang S, Wang Y, Rahman M, Syk I, et al. (2014) Proinflammatory role of neutrophil extracellular traps in abdominal sepsis. Am J Physiol Lung Cell Mol Physiol 307(7): L586-L596.

23. Butcher EC (1991) Leukocyte-endothelial cell recognition: three (or more) steps to specificity and diversity. Cell 67(6): 1033-1036.

24. Carlos TM, Harlan JM (1994) Leukocyte-endothelial adhesion molecules. Blood 84(7): 2068-2101

25. Vestweber D, Blanks JE (1999) Mechanisms that regulate the function of the selectins and their ligands. Physiol Rev 79(1): 181-213.

26. Wang Y, Du F, Hawez A, Morgelin M, Thorlacius H (2019) Neutrophil extracellular trap-microparticle complexes trigger neutrophil recruitment via high-mobility group protein 1 (HMGB1)-toll-like receptors (TLR2)/TLR4 signalling. Br J Pharmacol 176(17): 3350-3363.

27. Zuo Y, Yalavarthi S, Shi H, Gockman K, Zuo M, et al. (2020) Neutrophil extracellular traps in COVID-19. JCI Insight 5(11): e138999.

28. Earhart AP, Holliday ZM, Hofmann HV, Schrum AG (2020) Consideration of dornase alfa for the treatment of severe COVID-19 acute respiratory distress syndrome. New Microbes New Infect 35: 100689.

29. Saffarzadeh M, Juenemann C, Queisser MA, Lochnit G, Barreto G, et al. (2012) Neutrophil extracellular traps directly induce epithelial and endothelial cell death: a predominant role of histones. PLoS One 7(2): e32366.

30. Caudrillier A, Kessenbrock K, Gilliss BM, Nguyen JX, Marques MB, et al. (2012) Platelets induce neutrophil extracellular traps in transfusionrelated acute lung injury. J Clin Invest 122(7): 2661-2671.

31. Lv X, Wen T, Song J, Xie D, Wu L, et al. (2017) Extracellular histones are clinically relevant mediators in the pathogenesis of acute respiratory distress syndrome. Respir Res 18(1): 165

32. Lefrancais E, Mallavia B, Zhuo H, Calfee CS, Looney MR (2018) Maladaptive role of neutrophil extracellular traps in pathogen-induced lung injury. JCI Insight 3(3): e98178.

33. Liu S, Su X, Pan P, Zhang L, Hu Y, et al. (2016) Neutrophil extracellular traps are indirectly triggered by lipopolysaccharide and contribute to acute lung injury. Sci Rep 6: 37252

34. Narasaraju T, Yang E, Samy RP, Ng HH, Poh WP, et al. (2011) Excessive neutrophils and neutrophil extracellular traps contribute to acute lung injury of influenza pneumonitis. Am J Pathol 179(1): 199-210.

35. Wang Y, Luo L, Braun O0, Westman J, Madhi R, et al. (2018) Neutrophil extracellular trap-microparticle complexes enhance thrombin generation via the intrinsic pathway of coagulation in mice. Sci Rep 8(1): 4020 .

36. Zhou F, Yu T, Du R, Fan G, Liu Y, et al. (2020) Clinical course and risk factors for mortality of adult inpatients with COVID-19 in Wuhan, China: a retrospective cohort study. Lancet 395(10229): 1054-1062.

37. Guan WJ, Ni ZY, Hu Y, Liang WH, Ou CQ et al. (2020) Clinical Characteristics of Coronavirus Disease 2019 in China. N Engl J Med 382(18): 1708-1720.

38. Shak S, Capon DJ, Hellmiss R, Marsters SA, Baker CL (1990) Recombinant human DNase I reduces the viscosity of cystic fibrosis sputum. Proc Natl Acad Sci U S A 87(23):9188- 9192.

39. Dicker AJ, Crichton ML, Pumphrey EG, Cassidy AJ, Suarez Cuartin G, et al (2018) Neutrophil extracellular traps are associated with disease severity and microbiota diversity in patients with chronic obstructive pulmonary disease. J Allergy Clin Immunol 141(1): 117-127.

40. Manzenreiter R, Kienberger F, Marcos V, Schilcher K, Krautgartner WD, et al. (2012) Ultrastructural characterization of cystic fibrosis sputum using atomic force and scanning electron microscopy. J Cyst Fibros 11(2): 84-92.
41. Yaqian m, Lin W, Wen J, Chen G (2020) Clinical and pathological characteristics of 2019 novel coronavirus disease (COVID-19): a systematic review. medRxiv.

42. Nebbioso M, Livani ML, Santamaria V, Librando A, Sepe M (2018) Intracameral lidocaine as supplement to classic topical anesthesia for relieving ocular pain in cataract surgery. Int J Ophthalmol 11(12): 1932 1935.

43. Lv ZM, Chen L, Tang J (2011) Nebulized lidocaine inhalation in the treatment of patients with acute asthma. World J Emerg Med 2(1): 3032.

44. Caracas HC, Maciel JV, Martins PM, de Souza MM, Maia LC (2009) The use of lidocaine as an anti-inflammatory substance: a systematic review. J Dent 37(2): 93-97.

45. Eriksson AS, Sinclair R, Cassuto J, Thomsen P (1992) Influence of lidocaine on leukocyte function in the surgical wound. Anesthesiology 77(1): 74-78.

46. Negus RP (1996) The chemokines: cytokines that direct leukocyte migration. J R Soc Med 89(6): 312-314.

47. Nourshargh S, Alon R (2014) Leukocyte migration into inflamed tissues. Immunity 41(5): 694-707.

48. Flondor M, Listle H, Kemming GI, Zwissler B, Hofstetter C (2010) Effect of inhaled and intravenous lidocaine on inflammatory reaction in endotoxaemic rats. Eur J Anaesthesiol 27(1): 53-60.

49. Van Der Wal S, Vaneker M, Steegers M, Van Berkum B, Kox M, et al. (2015) Lidocaine increases the anti-inflammatory cytokine IL-10 following mechanical ventilation in healthy mice. Acta Anaesthesiol Scand 59(1): 47-55.

50. Ortiz MP, Godoy MC, Schlosser RS, Ortiz RP, Godoy JP, et al. (2016) Effect of endovenous lidocaine on analgesia and serum cytokines: doubleblinded and randomized trial. J Clin Anesth 35: 70-77.

51. Kuchalik J, Magnuson A, Tina E, Gupta A (2017) Does local infiltration analgesia reduce peri-operative inflammation following total hip arthroplasty? A randomized, double-blind study. BMC Anesthesiol 17(1): 63 .

52. Cone RA (2009) Barrier properties of mucus. Adv Drug Deliv Rev 61(2): 75-85.

53. Innes AL, Woodruff PG, Ferrando RE, Donnelly S, Dolganov GM, et al. (2006) Epithelial mucin stores are increased in the large airways of smokers with airflow obstruction. Chest 130(4): 1102-1108.

54. Lai SK, Wang YY, Wirtz D, Hanes J (2009) Micro- and macrorheology of mucus. Adv Drug Deliv Rev 61(2): 86-100.

55. Matthews LW, Spector S, Lemm J, Potter JL (1963) Studies on Pulmonary Secretions. I. The over-All Chemical Composition of Pulmonary Secretions from Patients with Cystic Fibrosis, Bronchiectasis, and Laryngectomy. Am Rev Respir Dis 88: 199-204.

56. Vasconcellos CA, Allen PG, Wohl ME, Drazen JM, Janmey PA, et al. (1994) Reduction in viscosity of cystic fibrosis sputum in vitro by gelsolin. Science 263(5149): 969-971.

57. Serra MF, Anjos Valotta EA, Olsen PC, Couto GC, Jurgilas PB, et al (2012) Nebulized lidocaine prevents airway inflammation, peribronchial fibrosis, and mucus production in a murine model of asthma. Anesthesiology 117(3): 580-591.

58. Galoș EV, Tat TF, Popa R, Efrimescu CI, Finnerty D, et al. (2020) Neutrophil extracellular trapping, and angiogenesis biomarkers after intravenous or inhalation anaesthesia with or without intravenous lidocaine for breast cancer surgery: a prospective, randomised trial. Brit J Anaesth 125(5): 712-721. 